\title{
Evaluation of High-Pressure Thermophysical Parameters of the Diacylglycerol (DAG) Oil Using Ultrasonic Waves
}

\author{
P. Kiełczyński ${ }^{1}$ - M. Szalewski ${ }^{1}$ - A. Balcerzak ${ }^{1}$ - K. Wieja ${ }^{1}$ • A. J. Rostocki ${ }^{2}$. \\ S. Ptasznik ${ }^{3}$
}

Received: 11 July 2016 / Accepted: 2 November 2016 /Published online: 16 November 2016

(C) The Author(s) 2016. This article is published with open access at Springerlink.com

\begin{abstract}
Modeling of high-pressure technological processes in the food industry requires knowledge of thermophysical parameters of processed foodstuffs in a broad range of pressures and temperatures. However, the high-pressure thermophysical parameters of foodstuffs are very rarely published in the literature. Therefore, further research is necessary to achieve a deeper insight into the biophysical and thermophysical phenomena under pressure to provide better control of technological processes and optimize the effects of pressure. The essential goal of this work is to evaluate the impact of high pressure and temperature on the thermophysical parameters of liquid foodstuffs on the example of diacylglycerol (DAG) oil (which attracted recently a considerable attention from research and industrial communities due to its remarkable benefits for health), using ultrasonic wave velocity and density measurements. Isotherms of adiabatic and isothermal compressibility, isobaric thermal expansion coefficient, internal pressure, and thermal pressure coefficient versus pressure were evaluated, based on the measurement of the compressional ultrasonic wave velocity and density of DAG oil at high pressures (up to $500 \mathrm{MPa}$ ) and at various temperatures. The adiabatic compressibility is affected mostly by the changes of pressure, i.e., it grows about four times when the pressure increases from the atmospheric
\end{abstract}

P. Kiełczyński

pkielczy@ippt.gov.pl

1 Institute of Fundamental Technological Research, Polish Academy of Sciences, ul. Pawińskiego 5B, 02-106 Warsaw, Poland

2 Faculty of Physics, Warsaw University of Technology, ul. Koszykowa 75, 00-662 Warsaw, Poland

3 Department of Fat and Meat Technology, Institute of Agricultural and Food Biotechnology, ul. Jubilerska 4, 04-190 Warsaw, Poland pressure $(0.1 \mathrm{MPa})$ to $400 \mathrm{MPa}$ at a temperature of $50{ }^{\circ} \mathrm{C}$. By contrast, the internal pressure is a pronounced function of the temperature, i.e., it increases six times when the temperature rises from 20 to $50{ }^{\circ} \mathrm{C}$ at a pressure of a $200 \mathrm{MPa}$. To perform numerical calculations, it was convenient to introduce a Tammann-Tait type equation of state to approximate the measured density isotherms of the investigated DAG oil. The results obtained in this paper can be applied in modeling and optimization of high-pressure technological processes and processing of foodstuffs. Evaluation of high-pressure isotherms of the considered thermophysical parameters of the DAG oil is an original authors' contribution to the state-ofthe-art.

Keywords High-pressure food processing • Diacylglycerols · Thermophysical parameters · Isothermal compressibility · Isobaric thermal expansion coefficient · Ultrasonic methods

\section{Introduction}

High-pressure food processing and conservation are nowadays fast developing technologies due to its numerous advantages. In fact, the high-pressure processing preserves the flavor, aroma, appearance, and color of the product without compromising its quality and nutritional characteristics (Rendueles et al. 2001). In addition, high-pressure technologies do not reduce the amount of vitamins in processed foods (Balasubramaniam et al. 2015; Bello et al. 2014, Barba et al. 2015). Moreover, the expiry date of many food products can be significantly extended after the high-pressure processing.

High-pressure technological processes of food preservation and processing are characterized by heat, mass, and momentum transfers occurring at various pressures and temperatures, as well as by possible phase transitions (LeBail et al. 2003). A 
sound understanding of pressure-dependent thermodynamic properties of foods is therefore crucial in designing of new products, equipment and technological processes (Min et al. 2010). The experimental evaluation of high-pressure physicochemical parameters of processed liquid foodstuffs is therefore vital for industrial developments.

The knowledge of thermophysical parameters of foods at high pressures is indispensable in development and optimization of mathematical models of food conservation and processing. For example, a number of thermophysical parameters of foods, such as their density, specific heat capacity, thermal expansion coefficient, and viscosity, as a function of pressure and temperature, must be known to model thermal profiles of pressurized food systems (Otero \& Sanz 2003; BarbosaCánovas \& Rodriguez 2005; Kowalczyk et al. 2005; Khurana \& Karwe 2009). Unfortunately, the data on the physicochemical parameters of foods at high-pressure range is not readily available (Guignon et al. 2012).

The primary objective of this work is to evaluate the impact of high pressures (up to $500 \mathrm{MPa}$ ) at various temperatures $\left(20-50^{\circ} \mathrm{C}\right)$ on thermophysical parameters of liquid foodstuffs on the example of diacylglycerol (DAG) oil (that is an important constituent of various fatty food products). To this end, we measured the speed of sound and density in samples of the DAG oil. The speed of sound of the DAG oil was measured using high-frequency ultrasonic waves propagating in the measured liquid. Ultrasonic measurement techniques are very convenient in practice since they are fast and nondestructive and can be fully automated and implemented online. By contrast, conventional methods used in estimation of thermophysical parameters of foods at high pressures are very cumbersome and difficult.

DAG-enriched oils have been drawing increased global attention from researchers and food manufacturers because of their health benefit. DAGs are natural components of numerous comestible oils (Shimizu et al. 2008). In addition to use as cooking oil, DAG oil has many other applications in fat-based food products, such as mayonnaises, salad dressings, margarines, shortenings, chocolates, and ice cream fats (Zhang et al. 2016). DAG oils have been widely used at different purity levels as additives enhancing fats' plasticity, emulsifiers and stabilizers in food, chemical, pharmaceutical, and cosmetic industries (Shimada \& Ohashi 2003; Camino Feltes et al. 2013; Satriana et al. 2016). Recent studies have shown that consumption of DAG oils results in metabolic characteristics that may be beneficial in preventing and managing obesity (Lo et al. 2008; Telle-Hansen et al. 2012, Ng et al. 2014).

The knowledge of thermophysical properties of liquids has a primordial relevance in design process in a number of industrial applications. Very important thermophysical parameters of liquids are the coefficient of thermal expansion $\alpha_{p}$ and isothermal compressibility $\beta_{T}$, since these parameters are frequently used in flow, heat transfer, and mass transfer calculations in many chemical and other industrial processes (Chorążewski \& Postnikov 2015). The compressibility is used to calculate the work done by compressional forces (Bridgman 1970).

On the other hand, the density is used to estimate compression heating of materials under pressure (Barbosa-Cánovas \& Rodriguez 2005) and to calculate other transport phenomena (Bird et al. 1976). Measurements of material volumetric properties under pressure are needed to address fundamentals of high-pressure processing engineering (Min 2008).

This paper presents the results of measurements of the speed of sound and density of the DAG oil at the pressure range $0-500 \mathrm{MPa}$, and temperatures from 20 to $50{ }^{\circ} \mathrm{C}$. The pressure $p$ and temperature $T$ dependence of the density was further approximated analytically by a Tammann-Tait type equation of state. As a consequence, the density of the DAG oil was expressed explicitly as a function of two variables, namely $p$ and $T$. Subsequently, we calculated the effect of pressure and temperature on the following crucial physicochemical parameters of the DAG oil, i.e., (a) isothermal compressibility $\beta_{T}$, (b) adiabatic compressibility $\beta_{S}$, (c) isobaric thermal expansion coefficient $\alpha_{p}$, (d) thermal pressure coefficient $\gamma$, and (e) internal pressure $P_{i n t}$. The numerical data obtained in this paper will be useful in optimization of highpressure technological processes in food processing and preservation industries. However, a further research is necessary to achieve a deeper insight into the biophysical and thermophysical phenomena occurring in pressurized liquids in order to provide better control of technological processes and their optimization (Guignon 2016). For example, it will be useful to extend the range of measurements in higher pressures and temperatures.

High-pressure thermophysical parameters of the DAG oil are very seldom published in the professional literature. In the present paper, the density $\rho(p, T)$ of the DAG oil was determined from measurements of the actual mass $\mathrm{m}$ and volume $V(p, T)$ of the measured liquid. On the other hand, the speed of sound $c(p, T)$ in the DAG oil was measured ultrasonically using high frequency $5 \mathrm{MHz}$ ultrasonic waves propagating in the measured DAG oil. Ultrasonic techniques have many advantages over conventional methods used in assessing the thermophysical parameters of liquid foodstuffs. In fact, the ultrasonic techniques are very convenient in practice since they are fast, noninvasive, and nondestructive and can be fully automated and implemented online. The ultrasonic waves generated in the investigated DAG oil are of relatively small amplitude (linear waves). Therefore, they did not affect thermodynamic properties of the measured liquid. By contrast, the conventional methods used in assessing the thermophysical parameters of liquid foodstuffs are cumbersome and difficult in implementation. They are rather laboratory methods, which cannot be used online. The experimental setup developed by 
the authors allows for a simultaneous measurement of the speed of sound and changes in the density of liquids at a broad range pressures at various temperatures.

The determination of the considered thermophysical parameters of the DAG oil, from measurements of the speed of sound and density in a wide range of pressures and temperatures, is an original authors' contribution to the state-of-theart.

\section{Material and Methods}

\section{Properties of the Measured DAG Oil}

DAGs are glycerides consisting of two fatty acid chains covalently bonded to a glycerol molecule through ester linkages. DAGs are esters of the trihydric alcohol glycerol in which two of the hydroxyl groups are esterified with long chain fatty acids.

The subject of the experiment were samples of DAG oil composed of $82 \%$ of DAGs and $18 \%$ of triacylglycerols (TAGs), with an extra vestigial content of monoacylglycerols (MAGs) and free fatty acids, see Table 1.

The composition of the measured DAQ oil was precisely determined using the gas chromatography AOCS Cd 11b-91 method, with a HP6890 device. The subsequent analysis was performed according to the ISO standards 5508 and 5509.

The main component of the DAG oil is an oleic acid C18:1 cis $(59.1 \%)$. The other significant components of the DAG oil are the following acids: linoleic C18:2 cis-cis (19.6\%), linolenic C18:3 cis-cis-cis (8.9\%), palmitic C16:0 (4.5\%),

Table 1 Fatty acid content of the investigated DAG oil sample according to ISO-5508 standard

\begin{tabular}{ll}
\hline Fatty acid (carbon atoms:C=C bonds) & Amount [\%] \\
\hline $\mathrm{C} 14: 0$ & 0.1 \\
$\mathrm{C} 16: 0$ & 4.5 \\
$\mathrm{C} 16: 1(\Sigma)$ & 0.5 \\
$\mathrm{C} 17: 0$ & 0.1 \\
$\mathrm{C} 17: 1$ & 0.1 \\
$\mathrm{C} 18: 0$ & 2.5 \\
$\mathrm{C} 18: 1(\Sigma c)$ & 59.1 \\
$\mathrm{C} 18: 2(c c)$ & 19.6 \\
$\mathrm{C} 18: 3(c c c)$ & 8.9 \\
$\mathrm{C} 20: 0$ & 1.9 \\
$\mathrm{C} 20: 1(\Sigma)$ & 1.0 \\
$\mathrm{C} 22: 0$ & 0.1 \\
$\mathrm{C} 22: 1(\Sigma)$ & 1.2 \\
$\mathrm{C} 24: 0$ & 0.3 \\
$\mathrm{C} 24: 1$ & 0.1 \\
\hline
\end{tabular}

stearic C18:0 (2.5\%), and arachidic C20:0 (1.9\%). Other fatty acids are presented in a small amount $(\leq 1.2 \%)$.

\section{Measurement Method}

High-pressure measurements were performed in the ultrasonic experimental setup designed and constructed by the authors, see Fig. 1. High-pressure conditions were achieved in a thickwalled cylindrical chamber (length $=23 \mathrm{~cm}$, diameter $=1.7 \mathrm{~cm}$, volume $=52 \mathrm{~cm}^{3}$ ) by forcing down a piston. The chamber was sealed by a Bridgman II sealing system, see Fig. 2.

The pressure inside the chamber was measured by a $100-\Omega$ manganin resistive sensor calibrated with a dead weight piston gauge of relative uncertainty $0.05 \%$. The resistance of the manganin sensor was measured and monitored using an NI 4065 multimeter PC card, provided by National Instruments Corp., Austin, TX, USA. The temperature in the chamber was kept constant within $\pm 0.01{ }^{\circ} \mathrm{C}$ using a precise thermostatic unit, manufactured by Julabo Labortechnik, Seelbach, Germany. A type T copper-constantan thermocouple was mounted inside the high-pressure chamber.

The speed of sound $c$ of ultrasonic waves propagating in the liquid was measured in a computerized setup especially designed to obtain a low level of the electrical noise and suppression of spurious ultrasonic signals. Special mounts for ultrasonic transducers were placed in the high-pressure chamber. The ultrasonic transducers were manufactured in-house using lithium niobate $\left(\mathrm{LiNbO}_{3}\right) 36^{\circ}$ Y-cut piezoelectric crystals, provided by Boston Piezo-Optics Inc., USA. The diameter of the piezoelectric crystal was $5 \mathrm{~mm}$ and its fundamental frequency $5 \mathrm{MHz}$. Two parallel surfaces of the crystal were metallized with a thin layer of chromium- and gold-forming transducer's electrodes. Piezoelectric transducers in the pressure chamber were facing each other and were in direct contact with the measured liquid.

The transmitting ultrasonic transducer was driven by a high power TB-1000 pulser-receiver PC card, provided by Matec, USA. The transmitting ultrasonic transducer was excited by a short tone burst electrical pulse with a RF frequency of $5 \mathrm{MHz}$, width $0.3 \mu \mathrm{s}$, and amplitude $300 \mathrm{~V}_{\mathrm{pp}}$. The pulse repetition period was $800 \mu$ s. Therefore, the ultrasonic signal was sent through the investigated liquid more than 1000 times per second. The ultrasonic signal detected by a receiving ultrasonic transducer was amplified and filtered in the Matec card and sent further to the PDA-1000 digitizer card, provided by Signatec, USA for digitization. Digitized ultrasonic signals with a sampling rate of $62.5 \mathrm{MHz}$ were further processed and stored inside an industrial PC computer. One value of the speed of sound $c$ in liquid was calculated from an averaged ultrasonic signal (1024 times). Signal averaging improves significantly signal-to-noise ratio of the received ultrasonic signal (32 times for 1024 averages). 
Fig. 1 Computerized ultrasonic experimental setup to measure the speed of sound $c$ and density $\rho$ of liquids at high pressures and various values of temperature

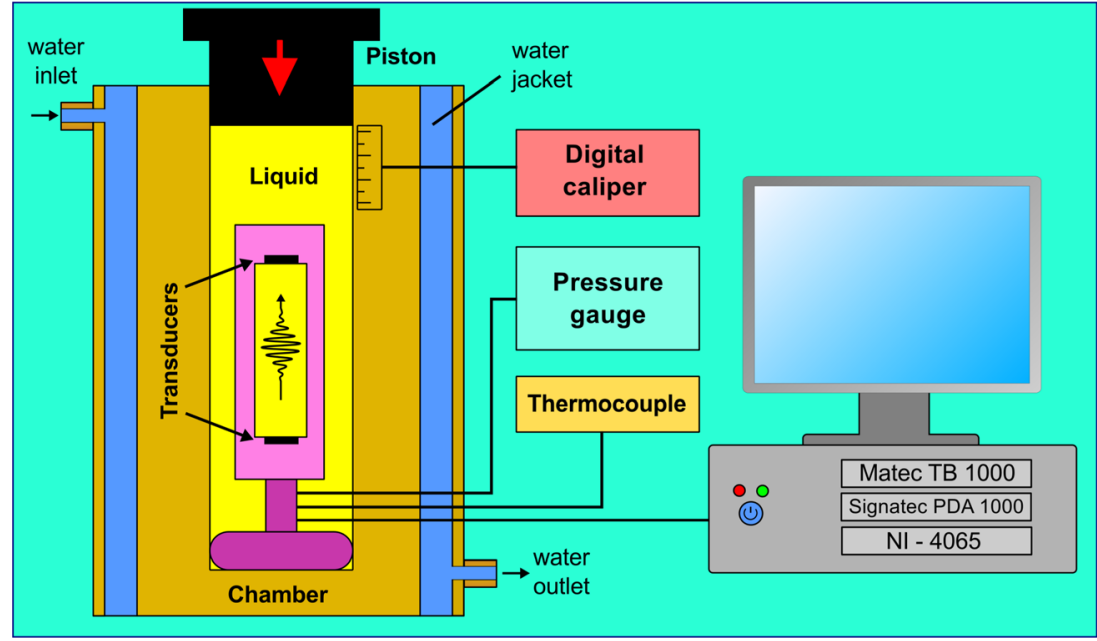

The time-of-flight (TOF) is the time for the ultrasonic signal to travel in the liquid from the sending transducer to the receiving transducer. The TOF between ultrasonic impulses traveling through the investigated liquid was determined using a cross-correlation method.

The computer program controlling operation of the PC cards (pulser-receiver ultrasonic card and digitizer card) as well as signal processing, data storage, and presentation was written with the Microsoft Visual C++ software package working in conjunction with the Measurement Studio software, provided by National Instruments. The PC cards and software were implemented within a Berta PC industrial computer, from Transduction, Mississauga, Canada.

The ultrasonic waves, generated in the measured liquid, were of longitudinal (compressional) type with the direction of particle vibrations parallel to the direction of wave propagation. The generated ultrasonic waves were of relatively small amplitude (linear waves). Therefore, they did not affect thermodynamic properties of the measured liquid. All measurements were carried out at precisely controlled temperatures, i.e., $T=20,30,40$, and $50{ }^{\circ} \mathrm{C}$.

\section{Measurements of Volume Changes}

Volume changes of the pressure chamber were determined from observations of the displacement of the piston mounted on the chamber's top. The displacement of the piston was measured by a digital caliper, see Fig. 1. Corrections resulting from the chamber expansion were evaluated from the Lamé equations and taken onto account during the final data analysis. The main source of error in determination of the actual volume of the pressure chamber was therefore the error in measurements of the piston displacement, which was measured by a digital caliper with a standard uncertainty $\sigma$ of
$0.02 \%$. Thus, the standard uncertainty $\sigma$ in determination of the chamber volume was estimated to be $0.06 \%$.

\section{Measurements of the Density}

The density of the DAG oil was measured initially at an atmospheric pressure (at various temperatures), using a $U$ shaped tube densitometer, provided by MG-2 UniLab, Poland. The density of the DAG oil at high-pressure conditions (up to $500 \mathrm{MPa}$ ) was evaluated by measuring changes in the volume occupied by the DAG oil sample, i.e., employing the following formula: $\rho(p, T)=m / V(p, T)$, where $m$ is the mass and $V(p, T)$ is the actual volume of the DAG oil sample in the chamber at a pressure $p$ and temperature $T$. Initial volume of the investigated DAG oil in the chamber at an atmospheric pressure was $22 \mathrm{~cm}^{3}$.

Since the mass $m$ of the DAG oil sample did not change during the experiment (no oil leak was observed), changes in the volume occupied by the DAG oil sample can be used to determine the density changes as a function of pressure. The standard uncertainty $\sigma$ for the density measurements was estimated to be $\pm 0.1 \%$.

\section{Measurements of the Speed of Sound}

The speed of sound $c$ for ultrasonic waves propagating in a liquid along a path $L$ is given by $c=L / t_{d}$ : where $t_{d}$ is the TOF along the path $L$. In the experimental setup, presented in Fig. 1, the TOF between two selected ultrasonic impulses was calculated using the cross-correlation method (Sugasawa 2002). Since the cross-correlation function can be efficiently calculated using fast Fourier transform (FFT) algorithms, the TOF between two ultrasonic impulses in the experimental setup from Fig. 1 was calculated in a few microseconds. 


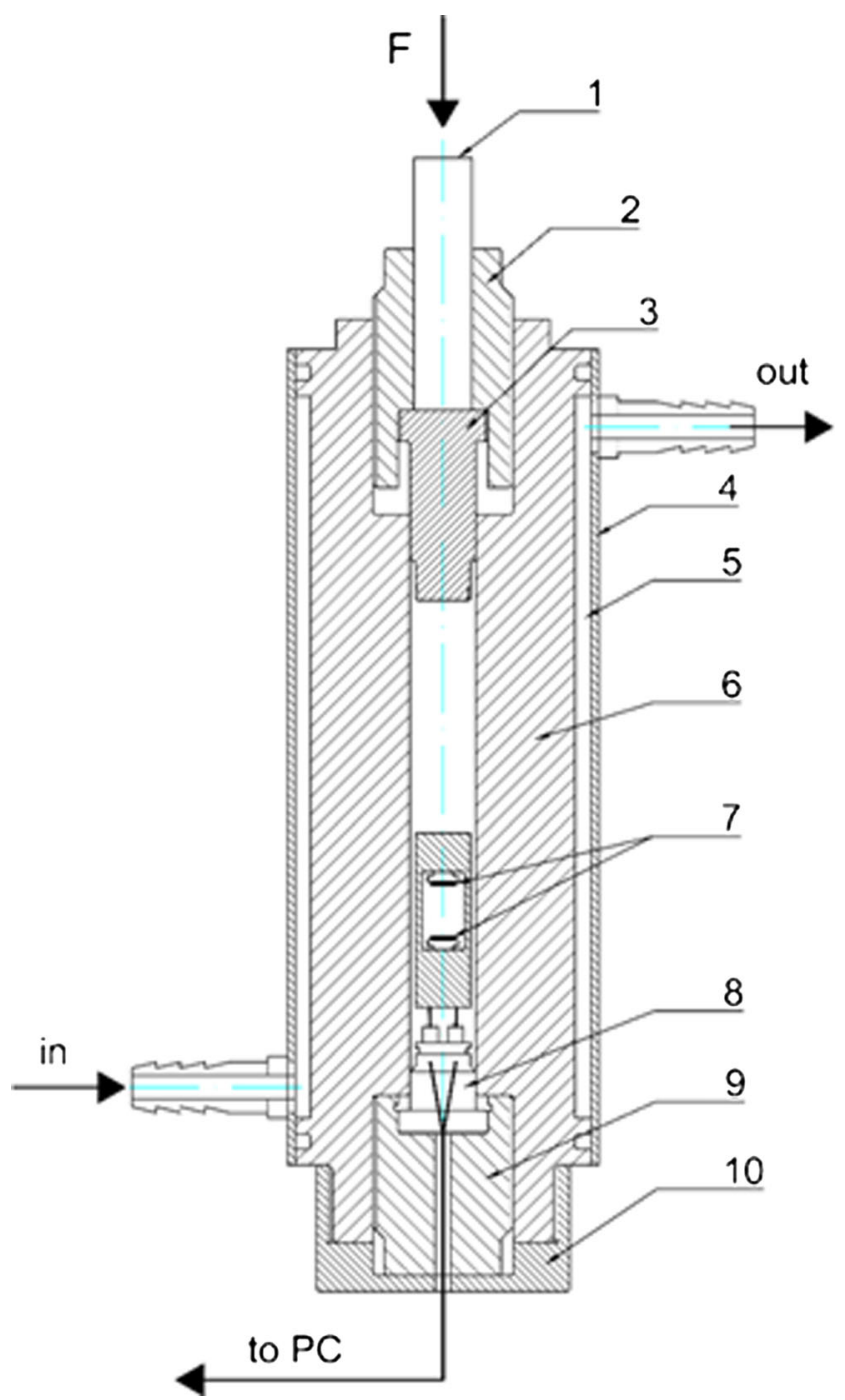

Fig. 2 Cross section of the high-pressure chamber used for measurements of thermophysical parameters of liquid foodstuffs. 1 piston extension, 2 upper plug, 3 high-pressure piston, 4 thermostatic jacket, 5 thermostatic fluid, 6 pressure chamber, 7 ultrasonic transducers, 8 electrical lead trough system for electrical wiring for pressure $p$ and temperature $T$ sensors and ultrasonic transducers, 9 lower plug, 10 base

According to the ISO guidelines (BIPM \& ISO 1995), the expanded $2 \sigma$ relative uncertainty for the speed of sound in liquid $\Delta U_{c} / U_{c}$ can be expressed as

$\frac{\Delta U_{c}}{U_{c}}=2 \sqrt{\left(\frac{\Delta U_{L}}{U_{L}}\right)^{2}+\left(\frac{\Delta U_{t_{d}}}{U_{t_{d}}}\right)^{2}}$

where $\Delta U_{L} / U_{L}$ is the relative standard uncertainty of the path $L$, and $\Delta U_{t_{d}} / U_{t_{d}}$ is the relative standard uncertainty of the TOF delay $t_{d}$.

Due to the employment of specialized signal processing procedures (FFT, cubic spline, interpolation, etc.), the TOF was measured with a picosecond resolution. However, due to extra systematic errors, resulting from pulse diffraction, signal distortions, etc., the resulting relative standard uncertainty $\Delta U_{t_{d}} / U_{t_{d}}$ for the TOF delay $t_{d}$ was estimated to be $\Delta U_{t_{d}} / U_{t_{d}}=0.1 \%$. Similarly, the standard uncertainty $\Delta U_{L} / U_{L}$ of the ultrasonic path $L$, resulting from calibration measurements in water, was estimated to be $\Delta U_{L} / U_{L}=0.1 \%$. As a result, the expanded $2 \sigma$ relative uncertainty for the measured speed of sound $c$ in liquid was estimated to be $0.3 \%$ (Kiełczyński et al. 2014). In other words, it was estimated that $95 \%$ of the measured results for the speed of sound in liquid will be located within a distance of $2 \sigma$ from the estimated value of the speed of sound.

\section{Results}

It has been discovered in the previous papers published by the authors (Kiełczyński et al. 2012a; Kiełczyński et al. 2012b; Rostocki et al. 2013b) that high-pressure phase transitions do occur in the DAG oil, olive oils and oleic acid (Rostocki et al. 2011, 2013a; Kiełczyński et al. 2014). Therefore, all measurements and computations, reported in this paper, were performed in the appropriate pressure range, starting from an atmospheric pressure up to the pressure at which phase transition begins, i.e., at $20{ }^{\circ} \mathrm{C}$ up to $220 \mathrm{MPa}$, at $30{ }^{\circ} \mathrm{C}$ up to $300 \mathrm{MPa}$, at $40{ }^{\circ} \mathrm{C}$ up to $380 \mathrm{MPa}$, and at $50{ }^{\circ} \mathrm{C}$ up to $520 \mathrm{MPa}$.

\section{Speed of Sound $c$ Measured in the DAG Oil}

Parameters of the ultrasonic wave, propagating in a liquid, are directly affected by the thermodynamic properties of the liquid, which in turn are functions of pressure and temperature (Winkelmeyer et al. 2016). Therefore, by measuring the speed of sound in liquid and ultrasonic attenuation, we can gain valuable information about the thermodynamic properties of the liquid. The speed of sound $c$ in liquid is directly associated with a number of thermodynamic properties of the liquid. Therefore, measurements of the speed of sound $c$ in the liquid and its density $\rho$, as a function of pressure $\mathrm{p}$ and temperature $T$, enable for the determination of a number of other thermodynamic properties of the liquid, such as adiabatic compressibility, isothermal compressibility, isobaric thermal expansion coefficient, etc. Figure 3 shows the speed of sound $c$ in the DAG oil measured as a function of pressure $p$ and temperature $T$.

As it is seen in Fig. 3, the speed of sound in the DAG oil decreases with the rise of temperature and increases with increasing pressures. The increase in the speed of sound $c$ can be attributed to a decrease in the intermolecular free path length and the adiabatic compressibility. 


\section{Sound Speed in DAG oil}

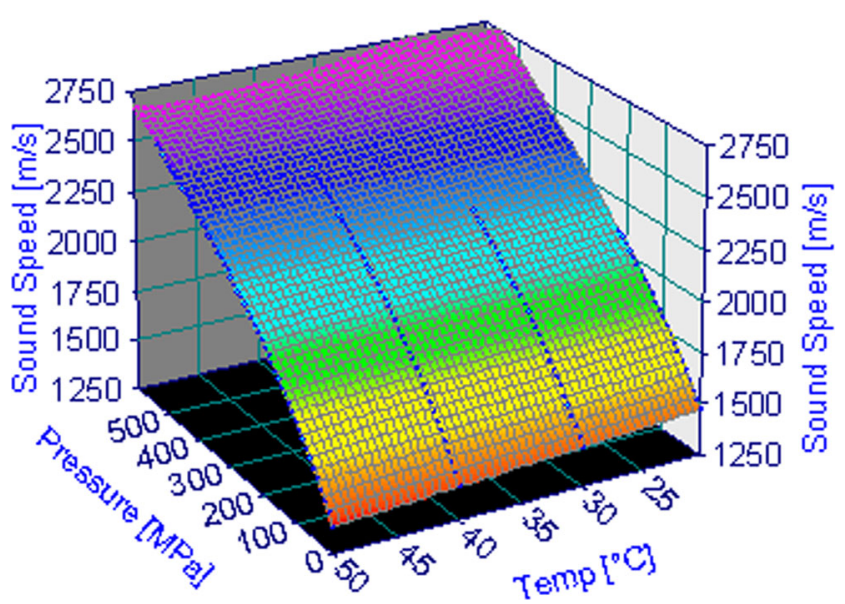

Fig. 3 Speed of sound $c$ in the DAG oil measured as a function of pressure and temperature at a frequency of $f=5 \mathrm{MHz}$.

\section{Density $\rho$ Measured in the DAG Oil}

The density of the DAG oil was measured at an atmospheric pressure for several different temperatures using a vibrating $\mathrm{U}$ tube densitometer, see Table 2.

Knowledge of the density of the processed liquid foodstuff versus pressure, at a constant temperature (isotherm), is crucial in engineering calculations and modeling of high-pressure technological processes of food processing and conservation. Figure 4 shows the results of measurements for the density of the DAG oil versus pressure and temperature. The compression of the DAG oil results in a smooth increase in its density as pressure grows. By contrast, the density of the DAG oil decreases with the temperature rise, as one might expected, taking into account the phenomenon of thermal expansion.

\section{Approximation of the DAG Oil Density by an Analytical Formula of the Tammann-Tait Type}

The density $\rho(p, T)$ of the DAG oil was measured for a finite number of pressures $p$ and temperatures $T$. However, we can determine the density $\rho$ at an arbitrary point $(p, T)$ using the appropriate analytical approximation formula. In this paper, the measured density isotherms of the DAG oil were approximated by an analytical function with two variables $(p, T)$, proposed by Tammann and Tait (Hayward 1967; Dymond \&

Table 2 Density of DAG oil sample measured at an atmospheric pressure at various temperatures

\begin{tabular}{lllll}
\hline Temp $\left[{ }^{\circ} \mathrm{C}\right]$ & 20 & 30 & 40 & 50 \\
\hline Density $\left[\mathrm{g} / \mathrm{cm}^{3}\right]$ & 0.93017 & 0.92324 & 0.91623 & 0.90911 \\
\hline
\end{tabular}

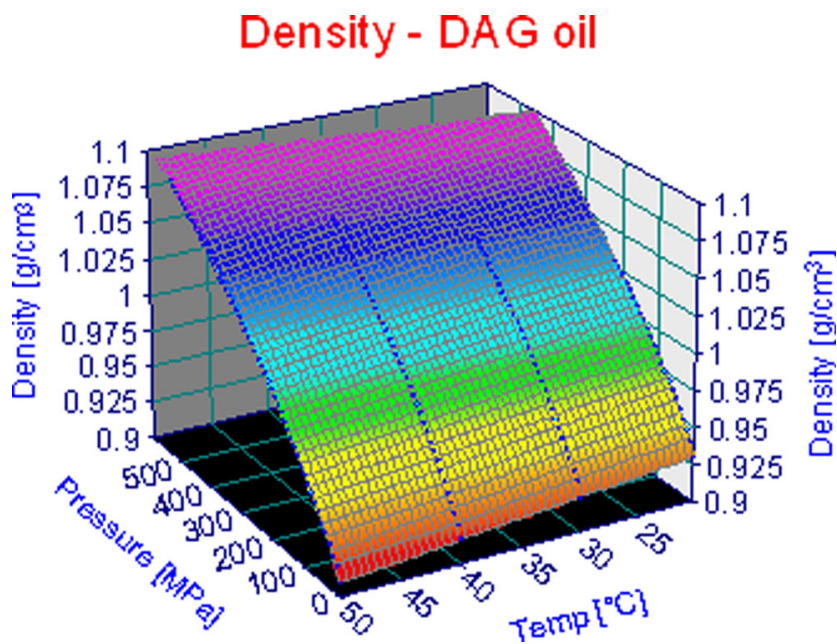

Fig. 4 Density $\rho$ of the DAG oil measured as a function of pressure and temperature

Malhotra 1988). The approximation function is as follows:

$$
\rho(p, T)=\frac{\rho_{0}+A_{3}\left(T-T_{0}\right)+A_{4}\left(T-T_{0}\right)^{2}}{1-A_{0} \ln \left(\frac{A_{1} e^{-A_{2}\left(T-T_{0}\right)}+p}{A_{1} e^{-A_{2}\left(T-T_{0}\right)}+p_{0}}\right)} \quad\left[\mathrm{g} / \mathrm{cm}^{3}\right]
$$

where $T_{0}$ is the reference temperature (in our case $20^{\circ} \mathrm{C}$ ), $p_{0}$ is the reference pressure $0.1 \mathrm{MPa}, \rho_{0}$ is the density at $\left(p_{0}, T_{0}\right)$ in grams per cubic centimeter, and $p$ is the actual pressure in megapascal.

Equation 2 depends on two variables $(p, T)$ and contains five free parameters $A_{0}, A_{1}, A_{2}, A_{3}$, and $A_{4}$, which have to be fitted to the experimental data for density. In this paper, the fitting process was performed using a TableCurve $3 \mathrm{D}$ version 4.0 (Systat, Richmond, CA, USA) software package. The numerical values of the adjusted parameters $A_{0}, A_{1}, A_{2}, A_{3}$, and $A_{4}$ are shown in Table 3.

The analytical expression, Eq. 2 with the five coefficients presented in Table 3, will be used in the following of "Thermophysical Parameters of the DAG Oil Calculated from the Measured Density $\rho$ and Speed of Sound $c$ " section to calculate a number of crucial physicochemical parameters of the DAG oil.

To assess the quality of approximation with the TammannTait empirical formula (Eq. 2), we have compared the results of measurements for the density of the DAG oil with the numerical values provided by the Tammann-Tait approximation. To quantify the difference between the measured and approximated densities, we calculated the following statistical parameters:

Table 3 Numerical values of the parameters $A_{0}, A_{1}, A_{2}, A_{3}$, and $A_{4}$ in Eq. 2 fitted to the measured DAG oil data

\begin{tabular}{llllll}
\hline $\mathrm{A}_{0}$ & $\mathrm{~A}_{1}$ & $\mathrm{~A}_{2}$ & $\mathrm{~A}_{3}$ & $\mathrm{~A}_{4}$ & $\mathrm{r}^{2}$ \\
\hline 0.12154 & 176.96429 & 0.00080433 & -0.00040716 & $-8.1415 \mathrm{e}-06$ & 0.9996
\end{tabular}

$r^{2}$ coefficient of determination 
the absolute average deviation (AAD), the maximum deviation (MD), the average deviation (Bias), and the standard deviation $\sigma$ that are defined as follows (Comuñas et al. 2008):

$$
\begin{aligned}
& \mathrm{AAD}=\frac{100}{N} \sum_{i=1}^{N}\left|\frac{\rho_{i}^{\text {exp }}-\rho_{i}^{\mathrm{cal}}}{\rho_{i}^{\text {exp }}}\right| \\
& \mathrm{MD}=\max \left(100\left|\frac{\rho_{i}^{\text {exp }}-\rho_{i}^{\mathrm{cal}}}{\rho_{i}^{\exp }}\right|\right) \\
& \mathrm{Bias}=\frac{100}{N} \sum_{i=1}^{N} \frac{\rho_{i}^{\text {exp }}-\rho_{i}^{\mathrm{cal}}}{\rho_{i}^{\exp }}
\end{aligned}
$$

$\sigma=\sqrt{\frac{\sum_{i=1}^{N}\left(\rho_{i}^{\exp }-\rho_{i}^{\text {cal }}\right)^{2}}{N-m}} \quad, \quad i=1, \ldots, \mathrm{N}$

where $\rho_{i}^{\exp }$ is the experimentally measured density, $\rho_{i}^{\text {cal }}$ represents the density calculated from the Tammann-Tait formula (Eq. 2), $N$ is the number of the experimental data $(N=145)$, and $m$ is the number of adjustable parameters in the Tammann-Tait equation (in our case $m=5$ ).

The statistical parameters of the Tammann-Tait approximation for the measured density of the DAG oil are given in Table 4 .

The obtained statistical parameters (AAD, MD, Bias, and $\sigma$ ) are much lower than the experimental uncertainty of the density measurements. Therefore, the values of the density calculated from the Tammann-Tait approximation formula (Eq. 2) are viable approximations of the measured density of the DAG oil.

Optimization procedures used in the approximation process are valuable and general tools that can be employed for many important applications in various domains of science and technology (Khasraghi et al. 2015; Valipour 2012a; Valipour 2012b; Valipour et al. 2015; Valipour 2016; Yannopoulos et al. 2015).

\section{Thermophysical Parameters of the DAG Oil Calculated from the Measured Density $\rho$ and Speed of Sound $c$}

\section{Isothermal Compressibility $\beta_{T}$}

The isothermal compressibility is a key physicochemical property of liquids in high-pressure technological processes, e.g., during food conservation under high-pressure (Barbosa 2003).

The isothermal compressibility $\beta_{T}$ is defined as follows:

$\beta_{T}(p, T)=\frac{1}{\rho(p, T)}\left(\frac{\partial \rho(p, T)}{\partial p}\right)_{T} \quad[1 / \mathrm{Pa}]$

Table 4 Statistical parameters of the Tammann-Tait approximation for the measured density of the DAG oil

\begin{tabular}{lllll}
\hline Statistical parameter & AAD [\%] & MD [\%] & Bias [\%] & $\sigma\left[\mathrm{g} / \mathrm{cm}^{3}\right]$ \\
\hline Numerical value & 0.021 & 0.11 & 0.0037 & $1.26 \times 10^{-4}$ \\
\hline
\end{tabular}

where $T$ is the temperature in Kelvin.

Using Eqs. 2 and 7, one obtains the following explicit formula for the isothermal compressibility:

$$
\beta_{T}(p, T)=\frac{A_{0}}{\left(A_{1} e^{-A_{2}\left(T-T_{0}\right)}+p\right)\left[1-A_{0} \ln \left(\frac{A_{1} e^{-A_{2}\left(T-T_{0}\right)}+p}{A_{1} e^{-A_{2}\left(T-T_{0}\right)}+p_{0}}\right)\right]}
$$

Figure 5 shows the isothermal compressibility $\beta_{T}$ of the DAG oil calculated with Eq. 8,

as a function of pressure, for various values of temperature.

The isothermal compressibility of the DAG oil grows with increasing temperatures and decreases with increasing pressures, see Fig. 5. The isothermal compressibility descends faster at lower pressures than at higher pressures.

\section{Adiabatic Compressibility}

The adiabatic compressibility is an important physical parameter of liquids. It depends on the structure of the liquid. Since the propagation of ultrasonic waves in liquids is an adiabatic process, the adiabatic compressibility $\beta_{S}$ can be calculated from the speed of sound as follows:

$\beta_{S}(p, T)=1 /\left(c^{2}(p, T) \times \rho(p, T)\right) \quad[1 / \mathrm{Pa}]$

where $c(p, T)$ is the speed of sound in liquid and $\rho(p, T)$ its density.

The dependence of the adiabatic compressibility $\beta_{S}$ of the DAG oil on pressure and temperature, calculated with Eq. 9, is shown in Fig. 6.

The adiabatic compressibility of the DAG oil, as a function of pressure and temperature, displays similar trends as the isothermal compressibility presented at Fig. 5.

\section{Isothermal Compressibility - DAG}

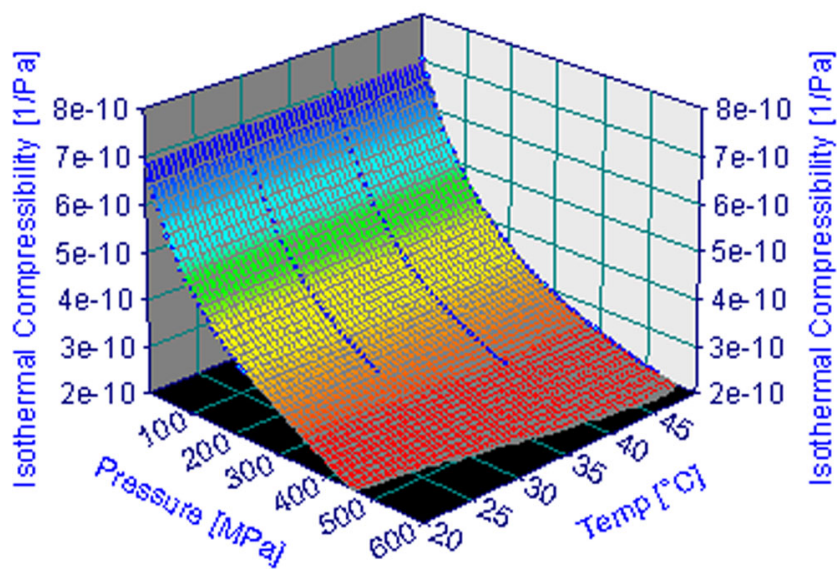

Fig. 5 Isothermal compressibility $\beta_{T}$ of the DAG oil as a function of pressure and temperature 


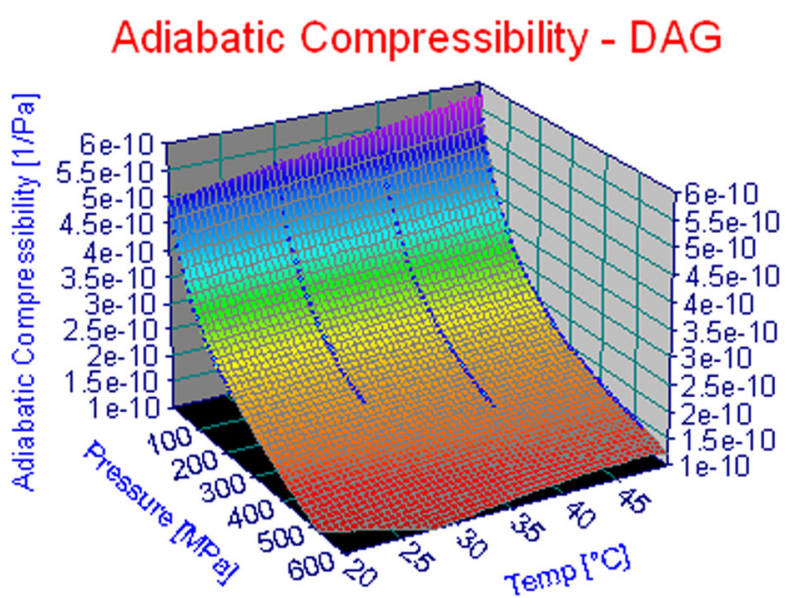

Fig. 6 Adiabatic compressibility $\beta_{S}$ of the DAG oil versus pressure and temperature

\section{Isobaric Thermal Expansion Coefficient}

The isobaric thermal expansion coefficient is a significant parameter used in design and control of high-pressure technological processes, e.g., in food industry (Aparicio et al. 2011).

The isobaric thermal expansion coefficient $\alpha_{p}$ is defined as follows:
Isobaric Thermal Expansion Coefficient - DAG

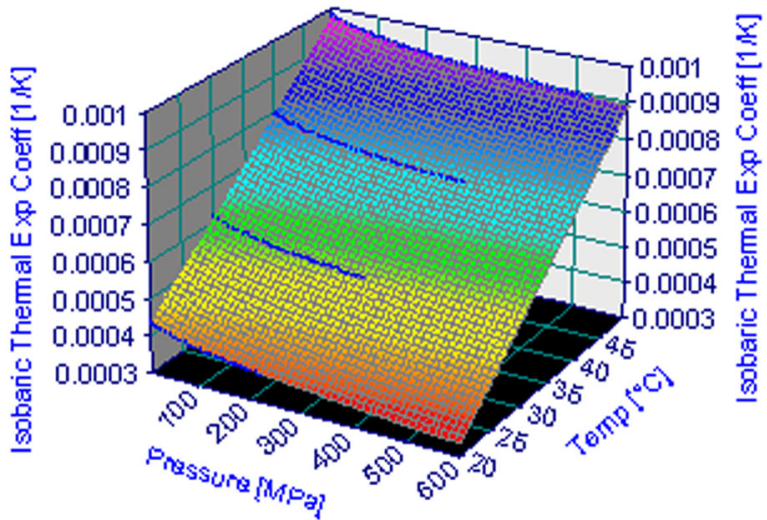

Fig. 7 Isobaric thermal expansion coefficient $\alpha_{p}$ of the DAG oil versus pressure and temperature

$$
\alpha_{p}(p, T)=\frac{-1}{\rho(p, T)}\left(\frac{\partial \rho(p, \mathrm{~T})}{\partial T}\right)_{p} \quad[1 / \mathrm{K}]
$$

Employing Eqs. 10 and 2, we arrive to the following formula for the isobaric thermal expansion coefficient:

$$
\begin{gathered}
\alpha_{p}(p, T)=\frac{A_{0} A_{1} A_{2} e^{-A_{2}\left(T-T_{0}\right)}\left(p-p_{0}\right)}{\left[1-A_{0} \ln \left(\frac{A_{1} e^{-A_{2}\left(T-T_{0}\right)}+p}{A_{1} e^{-A_{2}\left(T-T_{0}\right)}+p_{0}}\right)\right]\left(A_{1} e^{-A_{2}\left(T-T_{0}\right)}+p\right)\left(A_{1} e^{-A_{2}\left(T-T_{0}\right)}+p_{0}\right)} \\
-\frac{A_{3}+2 A_{4}\left(T-T_{0}\right)}{\rho_{0}+A_{3}\left(T-T_{0}\right)+A_{4}\left(T-T_{0}\right)^{2}}
\end{gathered}
$$

The dependence of the isobaric thermal expansion coefficient $\alpha_{p}$ of the DAG oil, on pressure and temperature, calculated with Eq. 11, is presented in Fig. 7.

Variations of the isobaric thermal expansion coefficient with pressure are complementary to variations of the isothermal compressibility with temperature (Barbosa 2003). Therefore, the isobaric thermal expansion coefficient of the DAG oil decreases with rising pressure. On the other hand, the values of the isobaric thermal expansion coefficient are positively affected by temperature, see Fig. 7.

\section{Thermal Pressure Coefficient $\gamma$}

The thermal pressure coefficient is one of the most fundamental parameters of liquids. It is closely related to various parameters of liquids, such as the isothermal compressibility, the speed of sound, the isobaric thermal expansion coefficient, the internal pressure, etc. (Moeini 2012).

The isochoric thermal pressure coefficient $\gamma$ of the DAG oil can be determined from the following formula:

$\gamma(p, T)=\frac{\alpha_{p}(p, T)}{\beta_{T}(p, T)} \quad[\mathrm{Pa} / \mathrm{K}]$

where $\alpha_{p}$ and $\beta_{T}$ stand, respectively, for the isobaric thermal expansion coefficient and the isothermal compressibility. Figure 8 shows the thermal pressure coefficient $\gamma$ of the DAG oil, evaluated from Eqs. 8, 11, and 12, as a function of pressure and temperature.

As it is shown in Fig. 8, the thermal pressure coefficient of the DAG oil grows monotonically as a function of pressure and temperature. 


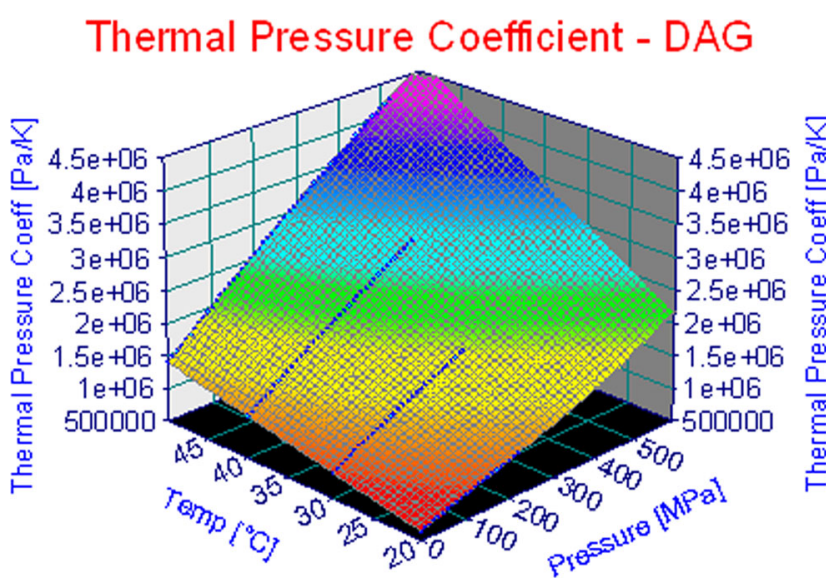

Fig. 8 Variations of the thermal pressure coefficient $\gamma$ of the DAG oil as a function of pressure and temperature

\section{Internal Pressure $P_{\text {int }}$}

The internal pressure is a very important and excellent tool to understand the nature of molecular interactions and internal structures of liquids (Polikhronidi et al. 2012).

The internal pressure $P_{\text {int }}$ can be calculated using the following equation (Perez \& Dayantis 1990):

$P_{\text {int }}(p, T)=\frac{T \alpha_{p}(p, T)}{\beta_{T}(p, T)}-p \quad[\mathrm{~Pa}]$

where $\alpha_{p}$ is the isobaric thermal expansion coefficient and $\beta_{T}$ the isothermal compressibility.

The plot of the internal pressure $P_{\text {int }}$ of the DAG oil as a function of pressure and temperature, calculated from the Eqs. 8, 11 and 13, is shown in Fig. 9.

The internal pressure of the DAG oil rises with increasing temperatures. The dependence of the internal pressure of the DAG oil on pressure is more complex. For lower temperatures, the internal pressure diminishes with increasing pressure. For higher temperatures, the internal pressure augments with increasing pressure, see Fig. 9.

\section{Discussion}

The values of thermophysical parameters of DAG oil change significantly with increasing temperature and pressure. For example, the adiabatic compressibility is affected mostly by the changes of pressure, i.e., it grows about four times when the pressure increases from the atmospheric pressure $(0.1 \mathrm{MPa})$ to $400 \mathrm{MPa}$ at a temperature of $50{ }^{\circ} \mathrm{C}$. By contrast, the internal pressure is a pronounced function of the temperature, i.e., it increases six times when the temperature rises from 20 to $50{ }^{\circ} \mathrm{C}$ at a pressure of a $200 \mathrm{MPa}$.

The main objective of this paper was to investigate the thermophysical properties of the DAG oil, such as its density,

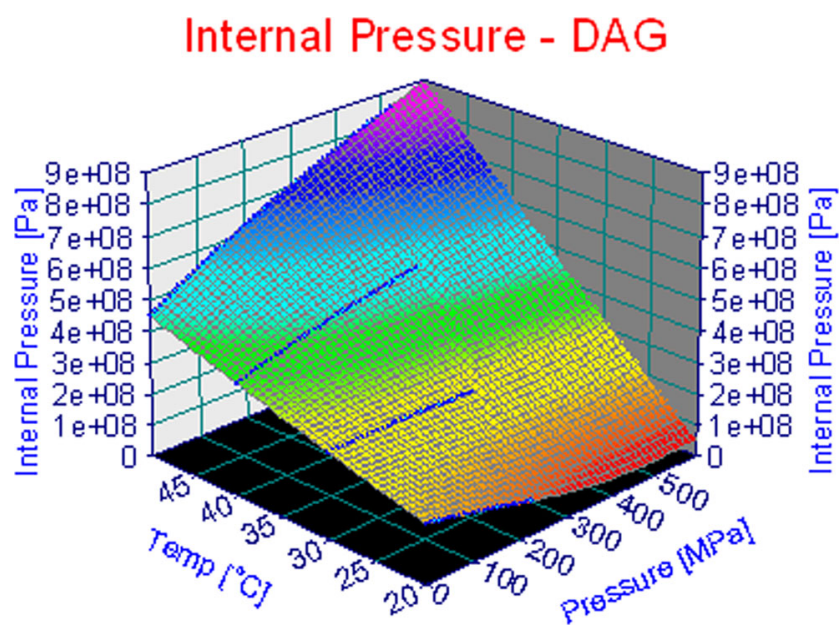

Fig. 9 The dependence of the internal pressure $P_{i n t}$ of the DAG oil versus pressure and temperature

compressibility, isobaric thermal expansion coefficient, thermal pressure coefficient, etc. Chemical properties of the DAG oil (although essential) were in fact out of scope of our research. In the range of applied pressures and temperatures, no phase transitions of the investigated liquid were observed. In this paper, we evaluated seven major thermophysical parameters of the DAG oil at high pressures for various temperatures. These parameters can be indispensable in modeling and design of the corresponding high-pressure equipment and technological processes (Sastry 2016; Norton \& Sun 2008; Otero \& Sanz 2003).

The data available in the literature shows that the effect of high pressures on the chemical structure of oils is rather of secondary importance in the range of pressures and temperatures applied in our investigations. At relatively low temperatures used in our measurements $\left(20-50{ }^{\circ} \mathrm{C}\right)$, the covalent bonds of oils are virtually unaffected. The limited effect of the high-pressure processing (HPP), at moderate temperatures, on covalent bonds represents a unique feature of this technology. In other words, the HPP has a minimal effect on food chemistry.

Similarly, the high-pressure processing has a negligible effect on compounds with low-molecular weight, such as flavor compounds, vitamins, and pigments by contrast to the thermal food processing. As a result, the quality of the HPP pasteurized food is virtually the same as that of fresh food products (Yordanov \& Angelova (2010); Balasubramaniam et al. 2015, Muntean et al. 2016; Kadam et al. 2012).

A characteristic feature of the HPP treatment is that the pressure alters mainly interatomic distances that are connected to weak interactions, for which bonding energy is distance dependent, such as van der Waals forces, electrostatic forces, or hydrogen bonding. As a consequence, a pressurized sample would preserve its strong bonding (mainly covalent bonds) unchanged. The above argumentation was essential in a statement that the biological activity of functional compounds, 
such as ascorbic acid, folates, antioxidants, and anthocyanins, is preserved under HPP treatment (Martinez-Monteagudo \& Saldaña 2014).

The changes in physicochemical properties of oils (triolein) under high-pressure conditions at room temperature were investigated in (Tefelski et al. 2010) using a Raman spectroscopy. The observed changes were mainly attributed to changes in the conformation of molecules. This phenomenon did not significantly affect the chemical properties of the investigated oils.

\section{Conclusions}

There is still a lack of sufficient information regarding changes of in situ thermophysical properties of foods during highpressure treatment. In situ thermophysical properties could be informative parameters that represent quality changes of food products in a pressurized environment (Park \& Jun 2015)

The essential aim of this study was to evaluate the influence of high pressure and temperature on the thermophysical parameters of liquid foodstuffs on the example of DAG oil (which is an important constituent of various fatty food products), using compressional ultrasonic wave velocity and density measurements. The use of ultrasonic methods (in contrast to conventional methods) greatly facilitates evaluation of the physicochemical parameters of foods under high pressure. Ultrasonic techniques have many advantages over conventional methods used in the food processing industry. In fact, the ultrasonic techniques are very convenient in practice since they are fast, noninvasive, and nondestructive and can be fully automated and implemented online. The ultrasonic waves generated in the investigated DAG oil were of relatively small amplitude (linear waves). Therefore, they did not affect thermodynamic properties of the measured liquid.

It has been found that the density is a very important physical property of liquids, from which a number of crucial thermophysical parameters of liquids (e.g., that of DAG oil) can be established. Approximation of the measured DAG oil density isotherms versus pressure by the Tammann-Tait type equation of state enabled the development of analytical formulas for such important thermophysical properties of the DAG oil, such as (a) the isothermal compressibility, (b) isobaric thermal expansion coefficient, (c) internal pressure, and (d) thermal pressure coefficient, as a function of pressure and temperature. Additionally, employing the compressional ultrasonic wave velocity measurements, the adiabatic compressibility isotherms were evaluated as a function of pressure. These data can be helpful in engineering practice to evaluate the optimum parameters of high-pressure technological processes in the food industry, without the necessity for complex and time consuming experimental investigations on the effects of temperature and high pressure on the thermophysical parameters of the processed food products.

Analytical formulas established in this work can be used in engineering calculations to design and optimize of highpressure technological processes of food conservation and processing, as well as to characterize high-pressure physicochemical properties of biofuels (Ivanis et al. 2016).

The determination of the considered thermophysical parameters of the DAG oil, from measurements of the speed of sound and density in a wide range of pressures and temperatures, is an original authors' contribution to the state-of-theart.

The authors hope that the results of research, presented in this paper, extended our knowledge about the high-pressure and high-temperature parameters of liquid foodstuffs and can be useful in the actual engineering practice in designing of new technological processes and systems in food industry.

\section{Compliance with Ethical Standards}

Conflict of Interest The authors declare that they have no conflict of interest.

Open Access This article is distributed under the terms of the Creative Commons Attribution 4.0 International License (http:// creativecommons.org/licenses/by/4.0/), which permits unrestricted use, distribution, and reproduction in any medium, provided you give appropriate credit to the original author(s) and the source, provide a link to the Creative Commons license, and indicate if changes were made.

\section{References}

Aparicio, C., Guignon, B., Otero, L., \& Sanz, P. D. (2011). Thermal expansion coefficient and specific heat capacity from sound velocity measurements in tomato paste from 0.1 up to $350 \mathrm{MPa}$ and as a function of temperature. Journal of Food Engineering, 104, 341347.

Balasubramaniam, V. M., Martinez-Monteagudo, S. I., \& Gupta, R. (2015). Principles and application of high pressure-based technologies in the food industry. Annual Review of Food Science and Technology, 6, 19.1-19.28.

Barba, F. J., Terefe, N. S., Buckow, R., Knorr, D., \& Orlien, V. (2015). New opportunities and perspectives of high pressure treatment to improve health and safety attributes of foods. A review. Food Research International, 77, 725-742.

Barbosa, R. D. (2003). High pressure and temperature dependence of thermodynamic properties of model food solutions obtained from in situ ultrasonic measurements. University of Florida. Ph.D. Dissertation.

Barbosa-Cánovas, G. V., \& Rodriguez, J. (2005). Thermodynamic aspects of high hydrostatic pressure food processing. In G. V. Barbosa-Cánovas, M. Tapia, \& M. Pilar Cano (Eds.), Novel food processing technologies. New York: CRC Press.

Bello, E. F. T., Martínez, G. G., Klotz Ceberio, B. F., Rodrigo, D., \& Martínez, L. A. (2014). High pressure treatment in foods. Foods, 3, 476-490. 
BIPM \& ISO (1995). Guide to the expression of uncertainty in measurement. Geneva: International Organization for Standardization ISBN 92-67-10188-9.

Bird, R. B., Stewart, W. E., \& Lightfoot, E. N. (1976). Transport phenomena. New York: Wiley.

Bridgman, P. W. (1970). The physics of high pressure. New York: Dover Publications.

Camino Feltes, M. M., de Oliveira, D., Block, J. M., \& Ninow, J. L. (2013). The production, benefits, and applications of monoacylglycerols and diacylglycerols of nutritional interest. Food Bioprocess Technology, 6, 17-35.

Chorążewski, M., \& Postnikov, E. B. (2015). Thermal properties of compressed liquids: experimental determination via an indirect acoustic technique and modeling using the volume fluctuations approach. International Journal of Thermal Sciences, 90, 62-69.

Comuñas, M. J. P., Bazile, J. P., Baylaucq, A., \& Boned, C. (2008). Density of diethyl adipate using a new vibrating tube densimeter from (293.15 to 403.15$) \mathrm{K}$ and up to $140 \mathrm{MPa}$. Calibration and measurements. Journal of Chemical and Engineering Data, 53, 986-994.

Dymond, J. H., \& Malhotra, R. (1988). The Tait equation: 100 years on. International Journal of Thermophysics, 9, 941-951.

Guignon, B. (2016). Food science and technology: preservation and processing below $50 \mathrm{MPa}$ and up to $1 \mathrm{GPa}$. In J. M. Recio, J. M. Menedez, \& A. O. de la Roza (Eds.), An introduction to highpressure science and technology (pp. 353-373). Boca Raton: CRC Press.

Guignon, B., Aparicio, C., Sanz, P. D., \& Otero, L. (2012). Orange juice pvT-properties for high pressure processing and modeling purposes: importance of soluble solids concentration. Food Research International, 46, 83-91.

Hayward, A. T. J. (1967). Compressibility equations for liquids: a comparative study. British Journal of Applied Physics, 18, 965-977.

Ivanis, G. R., Radovic, I. R., Veljkovic, V. B., \& Kijevcanin, M. L. (2016). Biodiesel density and derived thermodynamic properties at high pressures and moderate temperatures. Fuel, 165, 244-251.

Kadam, P. S., Jadhav, B. A., Salve, R. V., \& Machewad, G. M. (2012). Review on the high pressure technology (HPT) for food preservation. Journal of Food Processing and Technology, 3(1000135), 5.

Khasraghi, M. M., Sefidkouhi, M. A. G., \& Valipour, M. (2015). Simulation of open- and closed-end border irrigation systems using SIRMOD. Archives of Agronomy and Soil Science, 61(7), 929-941.

Khurana, M., \& Karwe, M. V. (2009). Numerical prediction of temperature distribution and measurement of temperature in high hydrostatic food processor. Food and Bioprocess Technology, 2, 279-290.

Kiełczyński, P., Szalewski, M., Balcerzak, A., Malanowski, A., Siegoczyński, R. M., \& Ptasznik, S. (2012a). Investigation of high-pressure phase transition in DAG (diacylglycerol) oil using the Bleustein-Gulyaev ultrasonic wave method. Food Research International, 49, 60-64.

Kiełczyński, P., Szalewski, M., Balcerzak, A., Malanowski, A., \& Rostocki, A. J. (2012b). Application of the Bleustein-Gulyaev wave method for investigation of high-pressure phase transitions in diacylglycerol oil (pp. 511-514). Dresden: IEEE International Ultrasonic Symposium Proceedings.

Kiełczyński, P., Szalewski, M., Balcerzak, A., Wieja, K., Rostocki, A. J., Siegoczyński, R. M., \& Ptasznik, S. (2014). Application of ultrasonic wave celerity measurement for evaluation of physicochemical properties of olive oil at high pressure and various temperatures. LWT-Food Science and Technology, 57, 253-259.

Kowalczyk, W., Hartmann, C., Luscher, C., Pohl, M., Delgado, A., \& Knorr, D. (2005). Determination of thermophysical properties of foods under high hydrostatic pressure in combined experimental and theoretical approach. Innovative Food Science and Emerging Technologies, 6, 318-326.
LeBail, A., Boillereaux, L., Davenel, A., Hayert, M., Lucas, T., \& Monteau, J. Y. (2003). Phase transitions in foods: effect of pressure and methods to assess or control phase transition. Innovative Food Science and Emerging Technologies, 4, 15-24.

Lo, S. K., Tan, C. P., Long, K., Yusoff, M. S. A., \& Lai, O. M. (2008). Diacylglycerol oil-properties, processes and products. A review. Food and Bioprocess Technology, 1, 223-233.

Martinez-Monteagudo, S. I., \& Saldaña, M. D. A. (2014). Chemical reactions in food systems at high hydrostatic pressure. Food Engineering Reviews, 6(4), 105-127.

Min S. (2008). Properties of food and buffer solutions during high pressure processing: in-situ measurement of density, compressibility, electrical conductivity and reaction volume. Dissertation, Graduate School of the Ohio State University.

Min, S., Sastry, S. K., \& Balasubramaniam, V. M. (2010). Compressibility and density of select liquid and solid foods under pressures up to $700 \mathrm{MPa}$. Journal of Food Engineering, 96, 568574.

Moeini, V. (2012). Calculation of thermal pressure coefficient of lithium fluid by pVT data. International Scholarly Research Network Physical Chemistry, 2012, 724230.11 pages

Muntean, M. V., Marian, O., Barbieru, V., Catunescu, G. M., Ranta, O., Drocas, I., \& Terhes, S. (2016). High pressure processing in food industry-characteristics and applications. Agriculture and Agricultural Science Procedia, 10, 377-383.

Ng, S. P., Lai, O. M., Abas, F., Kwong Lim, H. K., Beh, B. K., Ling, T. C., \& Tan, C. P. (2014). Compositional and thermal characteristics of palm olein-based diacylglycerol in blends with palm super olein. Food Research International, 55, 62-69.

Norton, T., \& Sun, D.-W. (2008). Recent advances in the use of high pressure as an effective processing technique in the food industry. Food and Bioprocess Technology, 1, 2-34.

Otero, L., \& Sanz, P. D. (2003). Modeling heat transfer in high pressure food processing: a review. Innovative Food Science and Emerging Technologies, 4, 121-134.

Park, S. H., \& Jun, S. (2015). Practical estimation of the in situ physical properties of foods under high pressure. Food Science and Biotechnology, 24, 777-782.

Perez, A., \& Dayantis, J. (1990). Compressibilities and limiting internal pressures from the Tammann-Tait empirical equation of state. Journal of Applied Physics, 68, 4330-4331.

Polikhronidi, N. G., Batyrova, R. G., Abdulagatov, I. M., Stepanov, G. V., \& Wu, J. T. (2012). PVT and thermal-pressure coefficient measurements of diethyl ether (DEE) in the critical and supercritical regions. Journal of Chemical Thermodynamics, 53, 67-81.

Rendueles, E., Omer, M. K., Alvseike, O., Alonso-Calleja, C., Capita, R., \& Prieto, M. (2001). Microbiological food safety assessment of high hydrostatic pressure processing: a review. $L W T$-Food Science and Technology, 44, 1251-1260.

Rostocki, A. J., Siegoczyński, R. M., Kiełczyński, P., Szalewski, M., Balcerzak, A., \& Zduniak, M. (2011). Employment of a novel ultrasonic method to investigate high pressure phase transitions in oleic acid. High Pressure Research, 31, 334-338.

Rostocki, A. J., Tarakowski, R., Kiełczyński, P., Szalewski, M., Balcerzak, A., \& Ptasznik, S. (2013a). The ultrasonic investigation of phase transition in olive oil up to $0.7 \mathrm{GPa}$. Journal of American Oil Chemists' Society, 90, 813-818.

Rostocki, A. J., Malanowski, A., Tarakowski, R., Szlachta, K., Kiełczyński, P., Szalewski, M., Balcerzak, A., \& Ptasznik, S. (2013b). The sound velocity measurement in diacylglycerol oil under high pressure. High Pressure Research, 33, 172-177.

Sastry, S. K. (2016). Toward a philosophy and theory of volumetric nonthermal processing. Journal of Food Science, 81(6), E1431-E1436.

Satriana, Arpi, N., Lubis, Y. M., Adisalamun, SUpardan, M. D., \& Mustapha, W. A. W. (2016). Diacylglycerol-enriched oil production 
using chemical glycerolysis. European Journal of Lipid Science and Technology, 118. doi:10.1002/ejlt.201500489.

Shimada, A., \& Ohashi, K. (2003). Interfacial and emulsifying properties of diacylglycerol. Food Science and Technology Research, 9, 142 147.

Shimizu, M., Kudo, N., Nakajima, Y., Matsuo, N., Katsuragi, Y., Tokimitsu, I., et al. (2008). Acidity and DAG content of olive oil recently produced on the Island of Mallorca. Journal of the American Oil Chemist Society, 85, 1051-1056.

Sugasawa, S. (2002). Time difference measurement of ultrasonic pulses using cross-correlation function between analytic signal. Japanese Journal of Applied Physics, 41, 3299-3307.

Tefelski, D. B., Jastrzębski, C., Wierzbicki, M., Siegoczyński, R. M., Rostocki, A. J., Wieja, K., \& Kościesza, R. (2010). Raman spectroscopy of triolein under high pressures. High Pressure Research, 30(1), 124-129.

Telle-Hansen, V. H., Narverud, I., Retterstøl, K., Wesseltoft-Rao, N., Mosdøl, A., Granlund, L., Forstrøm Christiansen, K., Lamglait, A., Halvorsen, B., Holven, K. B., \& Ulven, S. M. (2012). Substitution of TAG oil with diacylglycerol oil in food items improves the predicted 10 years cardiovascular risk score in healthy, overweight subjects. Journal of Nutritional Science, 1, 1-13.

Valipour, M. (2012a). Comparison of surface irrigation simulation models: full hydrodynamic, zero inertia, kinematic wave. Journal of Agricultural Science, 4(12), 68-74.
Valipour, M. (2012b). Sprinkle and trickle irrigation system design using tapered pipes for pressure loss adjusting. Journal of Agricultural Science, 4(12), 125-133.

Valipour, M. (2016). Optimization of neural networks for precipitation analysis in a humid region to detect drought and wet year alarms. Meteorological Applications, 23, 91-100.

Valipour, M., Sefidkouhi, M. A. G., \& Eslamian, S. (2015). Surface irrigation simulation models: a review. International Journal of Hydrology Science and Technology, 5(1), 51-70.

Winkelmeyer, C. B., Peyronel, F., Weiss, J., \& Marangoni, A. G. (2016). Monitoring tempered dark chocolate using ultrasonic spectrometry. Food and Bioprocess Technology. doi:10.1007 /s11947-016-1755-5.

Yannopoulos, S. I., Lyberatos, G., Theodossiou, N., Li, W., Valipour, M., Tamburrino, A., \& Angelakis, A. N. (2015). Evolution of water lifting devices (pumps) over the centuries worldwide. Water, 7 , 5031-5060.

Yordanov, D. G., \& Angelova, G. V. (2010). High pressure processing for foods preserving. Biotechnology and Biotechnological Equipment, 24(3), 1940-1945.

Zhang, N., Yang, X., Fu, J., Chen, Q., Song, Z., \& Wang, Y. (2016). Production of diacylglycerol-enriched oil by glycerolysis of soybean oil using a bubble column reactor in a solvent-free system. Journal of Oleo Science. doi:10.5650/jos.ess15241. 